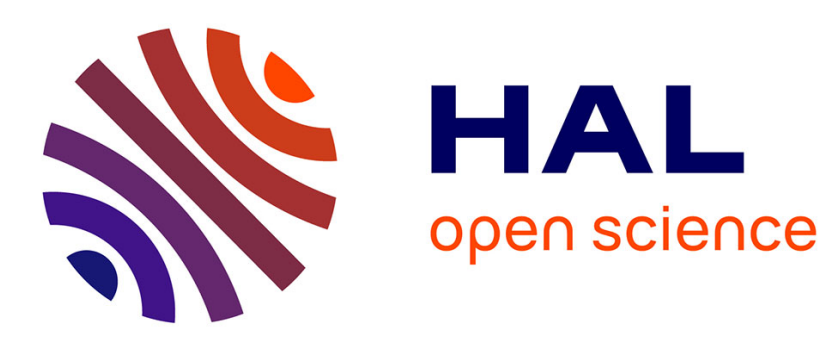

\title{
Three-boson relativistic bound states with zero-range two-body interaction
}

\author{
V.A. Karmanov, J. Carbonell
}

\section{To cite this version:}

V.A. Karmanov, J. Carbonell. Three-boson relativistic bound states with zero-range two-body interaction. European Conference on Few-Body Problems in Physics 18, Sep 2002, Bled, Slovenia. pp.417-418. in2p3-00012318

\section{HAL Id: in2p3-00012318 https://hal.in2p3.fr/in2p3-00012318}

Submitted on 23 Sep 2002

HAL is a multi-disciplinary open access archive for the deposit and dissemination of scientific research documents, whether they are published or not. The documents may come from teaching and research institutions in France or abroad, or from public or private research centers.
L'archive ouverte pluridisciplinaire HAL, est destinée au dépôt et à la diffusion de documents scientifiques de niveau recherche, publiés ou non, émanant des établissements d'enseignement et de recherche français ou étrangers, des laboratoires publics ou privés. 
Few-Body Systems Suppl. 0, 1-2 (2003)

Few-

Systemis

(c) by Springer-Verlag 2003

Printed in Austria

\title{
Three-boson relativistic bound states with zero-range two-body interaction
}

\author{
V.A. Karmanov ${ }^{1, *}$, J. Carbonell ${ }^{2, \dagger}$ \\ ${ }^{1}$ Lebedev Physical Institute, Leninsky Prospekt 53, 119991 Moscow, Russia \\ ${ }^{2}$ Institut des Sciences Nucléaires, 53 av. des Martyrs, 38026 Grenoble, France
}

Zero-range two-body interaction, applied to the nonrelativistic three-body system, results in the Thomas collapse [1]. The latter means that the threebody binding energy tends to $-\infty$ when the interaction radius tends to zero. Relativistic three-body calculations with zero-range interaction, performed in a minimal relativistic model [2] and in the framework of the Light-Front Dynamics [3], have shown that, due to relativistic repulsion, the three-body binding energy remains finite and the Thomas collapse is consequently avoided.

We have considered [4] in the field-theoretical framework, the problem of three equal mass $(m)$ bosons interacting via zero-range forces. Few points are worth to be precised.

(i) In quantum field theory the number of particles is not conserved. The three-body relativistic system is, therefore, an idealization. Our aim is to study whether this idealized relativistic system still exists for zero range interaction.

(ii) The vacuum remains bare (no vacuum fluctuations) only in the LightFront Dynamics. That is why we work in this approach.

(iii) In field theory all elementary interactions are point-like and, in this sens zero-range. We consider the Hamiltonian $H_{i n t}=\lambda \varphi^{4}(x)$ restricted to two-body system. It generates a two-body kernel which is simply the constant $\lambda$. This is the precise meaning of zero-range two-body interaction used in our study.

A relativistic Faddeev equation is derived, which provides a parameter-free relation between the two-body bound state mass $M_{2}$ and the three-body one $M_{3}$. It coincides with equation (11) from [3] except for the integration limits.

In figure 1(a) our calculations (solid line) of the three-body ground state mass $M_{3}$ as a function of the two-body one $M_{2}$ together with the dissociation threshold $M_{3}=M_{2}+m$ are displayed (in $m$ units). In the zero two-body binding limit $B_{2}=2 m-M_{2} \rightarrow 0$ the three-boson system has a binding energy

*E-mail address: karmanov@sci.lebedev.ru, karmanov@isn.in2p3.fr

$\dagger$ E-mail address: carbonel@isn.in2p3.fr 
$B_{3} \approx 0.012 \mathrm{~m}$. Dash line is the result obtained using the integration limits

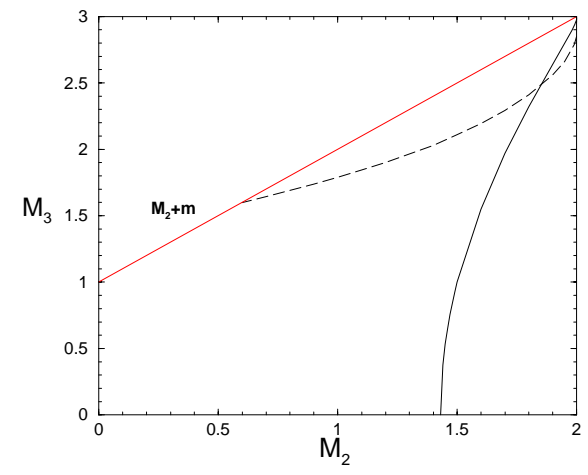

(a)

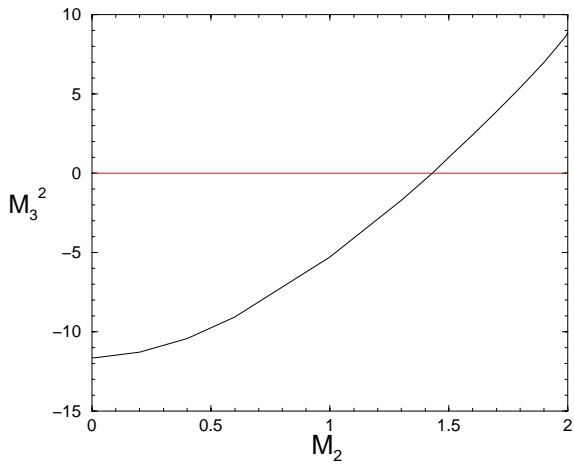

(b)

Figure 1. (a) Three-body bound state mass $M_{3}$ v.s. the two-body one $M_{2}$. Solid line corresponds to present work, dash line and dots - to calculations using the integration limits of [3]. (b) Three-body bound state mass squared $M_{3}^{2}$ v.s. $M_{2}$.

of [3]. In both cases, like in [2], the repulsive relativistic effects produce a natural cutoff, leading to a finite spectrum and - in the Thomas sense - an absence of collapse. However, the behaviours of the solid and dashed curves qualitatively differ from each other. When $M_{2}$ decreases, we found that the three-body mass $M_{3}$ (solid line) decreases very quickly and vanishes at the two-body mass value $M_{2}=M_{c} \approx 1.43 \mathrm{~m}$.

Results for $M_{3}^{2}$ are given in figure 1(b). For $M_{2} \leq M_{c}, M_{3}^{2}$ becomes negative and there are no physical solutions with real $M_{3}$ mass. However $M_{3}^{2}$ remains finite in all the two-body mass range $M_{2} \in[0,2]$ with $M_{3}^{2} \approx-(3.41 \mathrm{~m})^{2}$ in the limit $M_{2} \rightarrow 0$.

We conclude that in the system of three relativistic bosons with zero-range interaction, a relativistic counterpart of Thomas collapse takes place for strong enough two-body forces such that $M_{2}<M_{c}$. If in nonrelativistic treatment the system ceases to exist when its binding energy tends to $-\infty$, in the relativistic approach the system does not exist when its mass squared becomes negative.

\section{References}

1. L.H. Thomas, Phys. Rev. 47, 903 (1935).

2. J.V. Lindesay and H.P. Noyes, Preprint SLAC-PUB-2932 (1986).

3. T. Frederico, Phys. Lett. B282, 409 (1992).

4. J. Carbonell, V.A. Karmanov, nucl-th/0207073 (submitted for publication). 